\title{
Calibration and operation of an autonomous Water Cherenkov Detector using Red Pitaya in Quito
}

\author{
C. Rosero, J. Cando, N. Vásquez*, A. Carabalí and D. Erazo \\ Escuela Politécnica Nacional \\ E-mail: carlosrosero1326@gmail.com
}

\begin{abstract}
Escuela Politécnica Nacional (EPN), one of the institutes working in the field, developed some detectors based on Si-PMT and PMT. In this paper, we present the design of an autonomous water Cherenkov detector (WCD) capable of detecting astroparticles. The detector makes pulse digitization and calculates the rate of such particles arriving at $2850 \mathrm{~m}$ a.s.l. close to zero latitude. Using a commercial Red Pitaya board, the WCD registers temperature, pressure, and relative humidity at the site. The data from the conditioned PMT signal and from the environmental sensors are acquired and processed using a Red Pitaya board that digitizes the signal at $125 \mathrm{MHz}$. Additionally, taking advantage of a GPS precision, the collected information is synchronized. After 5 months of operation, we present the climate conditions in Quito and the temperature-pressure corrected fluxes of astroparticles. The development of this project has allowed the implementation of a low-cost monitoring station, providing useful information for the analysis of the behavior of the space weather.
\end{abstract}

36th International Cosmic Ray Conference -ICRC2019-

July 24th - August 1st, 2019

Madison, WI, U.S.A.

\footnotetext{
* Speaker.
} 


\section{Introduction}

Located on the Equator the Andean city of Quito offers a unique place to develop the field of astroparticle physics. In this work we present the status of the detector we built last year. Starting with a description on the geographical position of the detector, we continue with the results of the Corsika simulations, the description of the hardware and software setup, and finish with the discussion of the calibration process.

\section{Corsika Simulations}

Quito is located at: latitude $0^{0} 12^{\prime} 38^{\prime \prime} \mathrm{S}$ and longitude $78^{0} 29^{\prime} 20^{\prime \prime} \mathrm{W}$. Using Corsika software, we determine the fluxes of particles at the detector level. The models used were Quark Gluon String Jet II for high energy hadronic interactions[1] and Geisha for low energy hadronic interactions. The detector is located at $2850 \mathrm{~m}$ a.s.l., and the magnetic rigidity was calculated using the World Magnetic Model. We obtained a value of $26.885 \mathrm{nT}$ for the North Component and $10.166 \mathrm{nT}$ for the vertical component. We simulated the flux of particles at the detector lever for gammas and protons as primary particles with energies between $500 \mathrm{GeV}$ and $10 \mathrm{TeV}$. The results are shown in Figure 1.

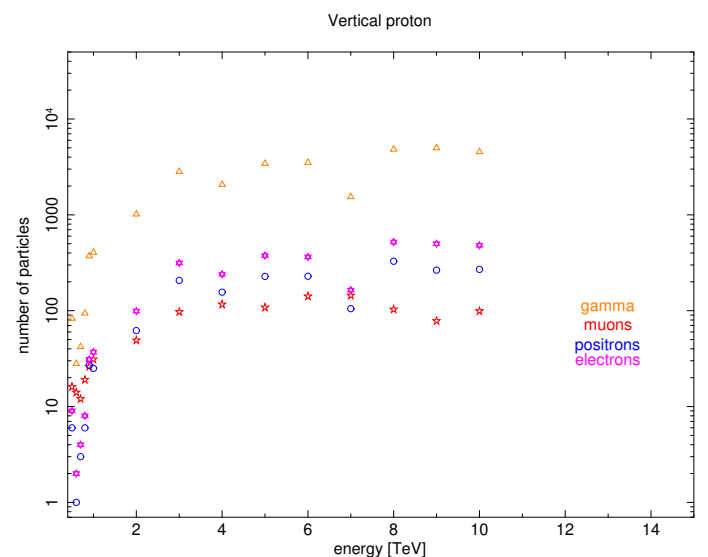

(a) Proton

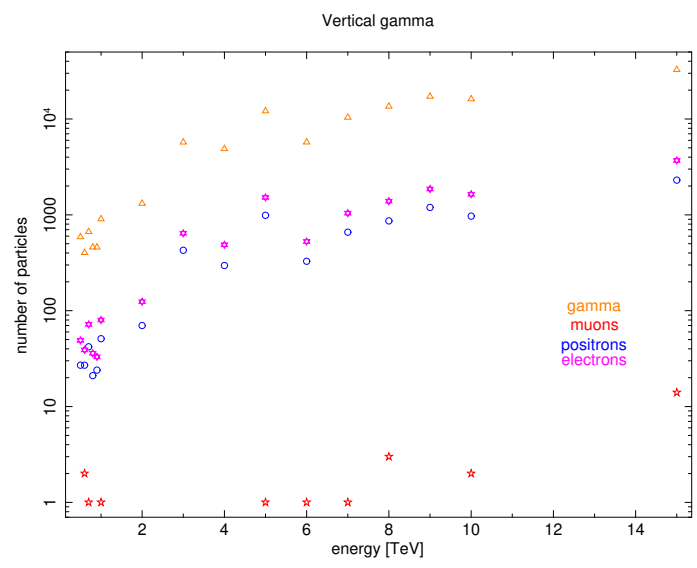

(b) Gamma

Figure 1: Corsika Simulations

\section{Hardware Setup}

The monitoring station has three important parts: a water Cherenkov detector (WCD), a data acquisition process and a solar power system. These components allow to have an autonomous detector that works for longer periods of time, and at high altitude where there is no power line distribution. On the other hand, the system could be replicated in other countries due to its easy construction and low cost. Figure 2(a) shows a block diagram where the subcomponents can be seen: a small electric cabinet that contains a voltage sources distribution board, Red Pitaya board, solid state disk (SSD), and a principal board used to attach the electronic elements. In a separate 
electric cabinet there is a solar controller connected to a battery and a solar panel located on the metal structure. There is also a plastic shell for environmental variables sensors and the WCD with one photomultiplier tube (PMT). In the Figure 2(b) we present the station implemented.

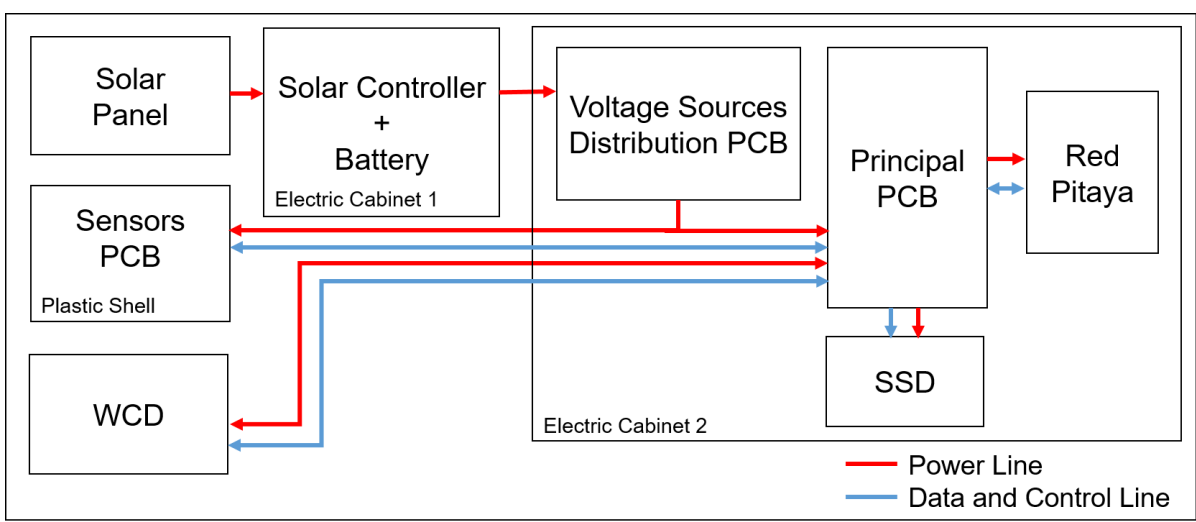

(a) Block diagram of monitoring station

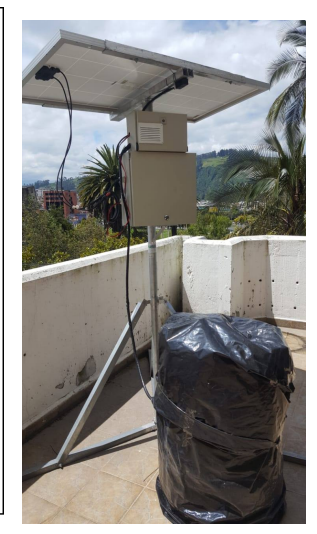

(b) Frontal view

Figure 2: Monitoring Station

The WCD uses a 9" PMT model XP1805 with 8 dynodes focused lineally, a sensitive window with borosilicate glass and a bi-alkali as photocathode. The PMT is connected to previous design from Pierre Auger Observatory. The Auger Base contains a high-voltage power module, a polarization circuit for the dynodes of the PMT, an amplifier circuit from the last dynode signal, coupling capacitors, an AD592 temperature sensor, coaxial cables for the outputs. The outputs are one from the anode signal and another from the amplified dynode signal and control cables with a DB15 terminal [2]. We performed several tests to determine the linear behavior between control variables and the output signals for example high voltage control and monitoring signal dependencies. These relationships were included in the software programming.

We used a plastic container for the WCD measuring $89[\mathrm{~cm}]$ in height and a $56[\mathrm{~cm}]$ in diameter, with a volume of 208.2 [liters]. The main modification done to the container was providing it with an optical isolation. The external surface of the container was painted with black enamel, in addition we covered it with three layers of aluminum foil. The inner surfaces have been covered by a high diffusive and reflective Tyvek, the dielectric material used in this station is water due to its low cost and technical availability and finally hydrogen peroxide was added to prevent the proliferation of bacterias in the water.

Ecuador has a high solar radiation all year long. An alternative and flexible option as an energy power supply method is the photovoltaic system. The sizing of all components is described in the IEEE 1562-2007 standard that uses a combination of the environmental characteristics of the geographical area and the energy requirements of the station. The "Peak Sun Hour" (PSH) method is used with an estimation of the average energy demand during a day[4]. The monitoring station requires several voltage levels; for this we used DC/DC converters in a buck topology. The design considers a primary source of $+12[\mathrm{~V}]$.

The variables that affect the detection of particles from the extensive air showers are temperature, pressure, and humidity. The effect that produces these variables in the measurement of 
astroparticles is analyzed in the next section. To be able to measure these variables we use robust sensors with high precision, low power consumption and low cost are required. We used a BMP180 sensor for temperature and atmospheric pressure measurements and a Si7021 sensor for humidity. Finally, the U-blox NEO-6M GPS module was selected for position and altitude measurements this device has a Pulse Per Second (PPS) signal used to synchronize information and a UART interface for communication.

For the implementation of the data acquisition process, the Red Pitaya board was used due to its high speed digital analog converters (125 MSPS) integrated into the same board. It also has different communication interfaces such as I2C, UART, USB that make it flexible to use with other peripherals. To connect the different stages of the system is necessary to prepare different monitoring and control signals. For this purpose we designed electronic boards with the necessary circuits. Figure 3 shows the connections that we made. Figure 3(a) presents the board designed with SMD elements and Figure 3(b) shows the connections of Red Pitaya. A complete description of the design and implementation of the detector can be found in the thesis by Cando and Rosero [5].

11

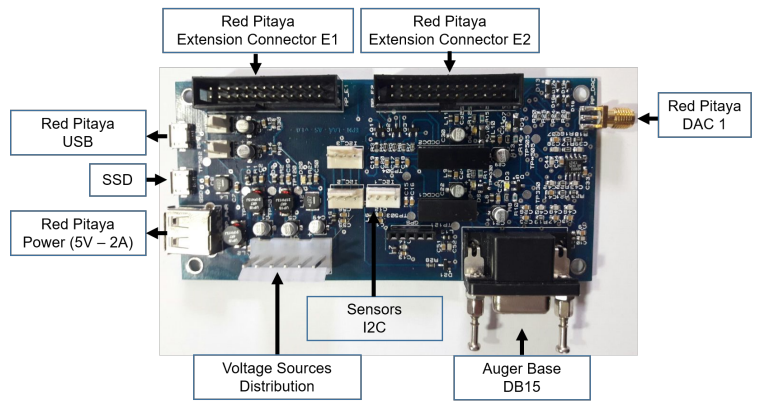

(a) Principal PCB connections

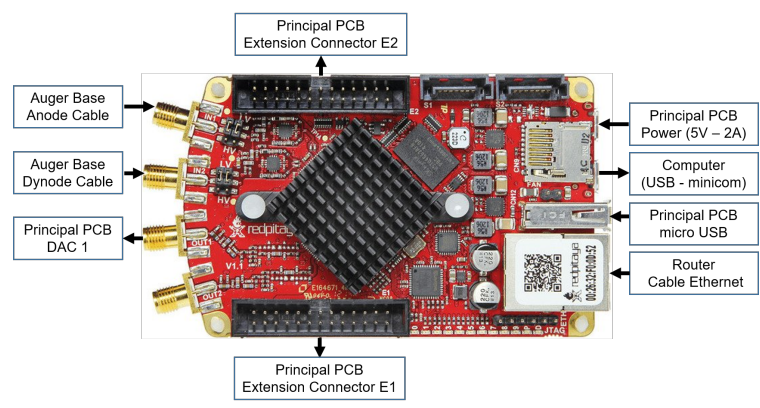

(b) Red Pitaya connections

Figure 3: Connections diagram

\section{Software Setup}

For the software design, we chose the Red Pitaya operative architecture which includes a Zynq7010. It has two sections: Processing System (PS) and Programmable Logic (PL). The processing system contains a Dual ARM Cortex-A9 processor as well as multiple digital communication peripherals and the static and dynamic memory interface controllers. In the programmable logic section we can reconfigure the hardware design of the FPGA using new algorithms in VHDL and Verilog languages.

We designed an application in C programming language to process the data in Red Pitaya. The diagram in Figure 4 shows the constituent parts of the device with its configuration files. The monitoring station has several devices which must be interpreted or controlled either through a communication protocol, digital or analog outputs/inputs such as ADC of 125 MSPS, XADC of 1 MSPS, DAC of 125 MSPS. Other peripherals are connected to the processing system such as I2C, UART, USB 2.0 communication systems. 


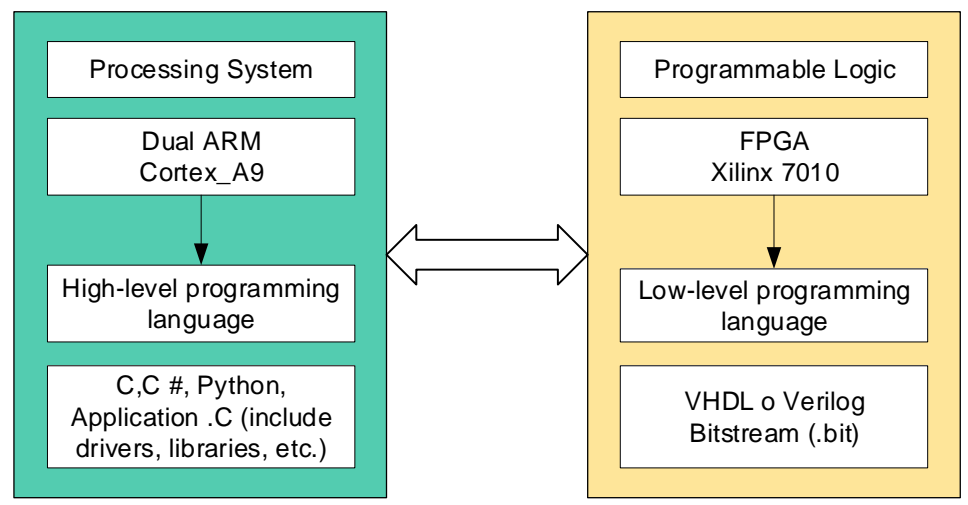

Figure 4: Internal architecture of Red Pitaya

The programming of Red Pitaya is done in such a way that it acquires, filters, and sends the information of the events from the FPGA to the processor. This is done with a high speed of data transfer in order to store it with the information acquired by the sensors and GPS from the processing system. Additionally, all information is synchronized with the PPS signal generated by the GPS, as shown in Figure 5. In this project we used different modules by default in Vivado, other modules created by programming developers for Red Pitaya as Pavel Demin [3] and modules designed exclusively to accomplish the objectives of the project.

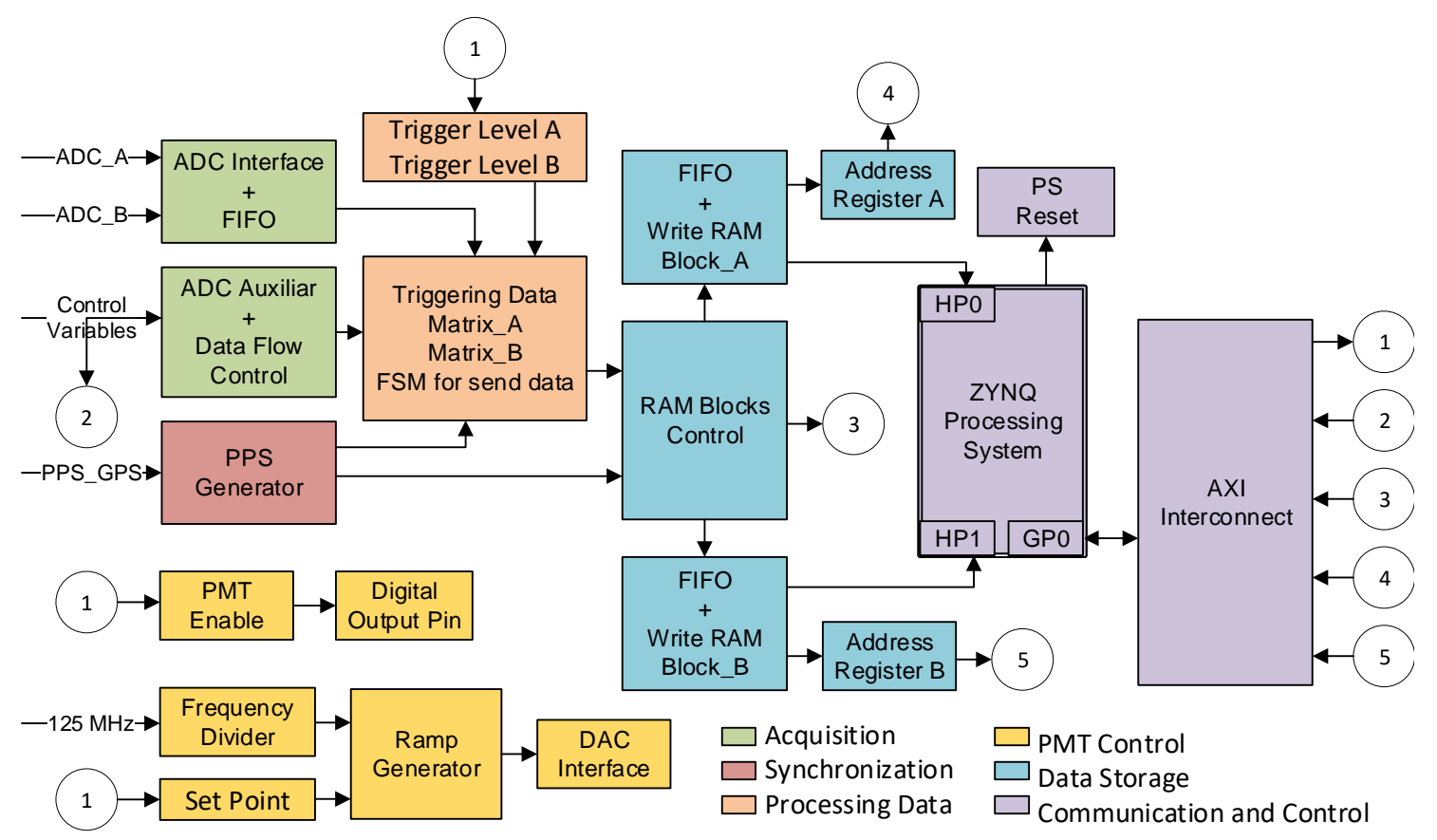

Figure 5: Simplified diagram of VHDL programming in Vivado

In figure 5 we see six parts, one is acquisition part where we obtained two inputs each of 14 bits at a frequency of $125 \mathrm{MHz}$. An integrated 12-bit XADC with a frequency of $1 \mathrm{MHz}$ is used 
to digitize the output signals of the Auger base and the FPGA. The same device is responsible for the configuration of the Dynamic Reconfiguration Port (DRP) mode and for reading the control variables in unipolar mode. The information generated by the FPGA and the data collected in the processing system is synchronized with the GPS time with a digital signal PPS. To obtain filtered data we process the component data of the ADC to $125 \mathrm{MHz}$ and the XADC to $1 \mathrm{MHz}$ together, the information is analyzed to obtain filtered data. Two data matrices are created: Matrix A with the information of filtered events by a trigger level and Matrix B with the information of the variables measured with auxiliary inputs of Red Pitaya, the latter is sent every second.

PMT control part generates a ramp signal in order to control the high voltage inside the PMT in an appropriate way. To reduce data storage loss during the data transfer we used finite state machine to switch between two blocks of RAM each with a capacity of 16 MB every one. Finally, the Communication and Control part necessary for the control in the transmission of data from the FPGA to the processing system, the General Purpose Ports (GP) ports and the High Performance Ports (HP) was implemented.

The Processing System uses a modified Debian Linux as operating system which is compatible with the algorithms in C-language, libraries for the BMP180 sensor and the data from the GPS. The program is designed to display a menu interface in which the user can control and enter the data collection input parameters such as the high voltage level in the PMT, the trigger level, the number of events and files that the user wants to save. The program consists of two processes, created in the main algorithm. The communication between these processes works through pipes, obtaining information from the environmental sensors every minute. Software generates and saves the acquired data in files on the SSD.

\section{Calibration}

The calibration of the sensors used in this project was performed in the calibration laboratories of the Instituto Nacional de Metereología e Hidrología (INAMHI), which is responsible for maintaining meteorological stations network in Ecuador. As a result, different adjustment equations were obtained to reduce the relative error in the measurements and to ensure information quality obtained by the station.

\section{Data Analysis}

The data acquired from August 2018 to May 2019 has particle rate $\left[\right.$ counts. $m^{-2} . s^{-1}$ ], relative humidity $[\%]$, temperature $\left[{ }^{\circ} \mathrm{C}\right]$, and atmospheric pressure $[\mathrm{hPa}]$. Data is on line, available in http://201.159.223/poli/index.php. The fluctuations observed in temperature during daytime reflect the location of the detector, situated on a terrace behind the building at $30[\mathrm{~m}]$ above the ground. The shadow of the building and the wind produce a variability of temperature measurements. We obtained a standard deviation for November which are divided in periods of 6 hours per day that starting at the middle of the day. Also we obtained the mean value of temperature, pressure, and humidity. There is a dependence between the atmospheric temperature and pressure; when the atmospheric temperature increases, the pressure decreases and the atmospheric depth increases in size letting particles cover longer distances in the atmosphere[7]. 

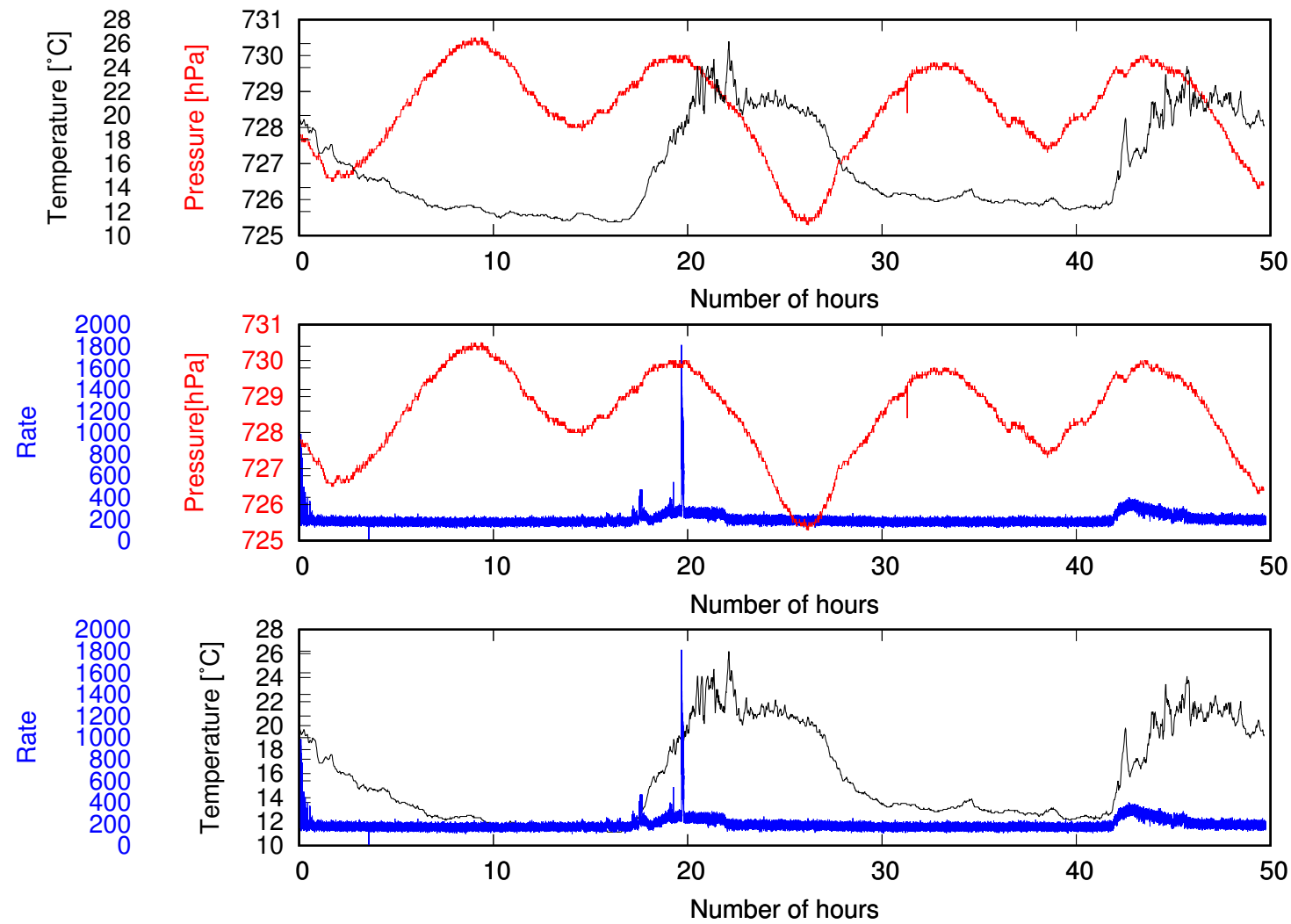

Figure 6: Samples of November data

\begin{tabular}{|c|c|c|c|c|c|c|c|c|c|}
\hline & $12 \mathrm{pm}-6 \mathrm{pm}$ & $6 \mathrm{pm}-12 \mathrm{am}$ & $12 \mathrm{am}-6 \mathrm{am}$ & $6 \mathrm{am}-12 \mathrm{pm}$ & $12 \mathrm{pm}-6 \mathrm{pm}$ & $6 \mathrm{pm}-12 \mathrm{am}$ & $12 \mathrm{am}-6 \mathrm{am}$ & $6 \mathrm{am}-12 \mathrm{pm}$ & $12 \mathrm{pm}-2 \mathrm{pm}$ \\
\hline Temperature & $15.72 \pm 1.84$ & $12.18 \pm 0.41$ & $11.66 \pm 0.60$ & $20.39 \pm 2.64$ & $18 \pm 2.87$ & $13.2 \pm 0.27$ & $12.63 \pm 0.30$ & $19.9 \pm 2.28$ & $19.96 \pm 0.68$ \\
\hline Pressure & $727.44 \pm 0.68$ & $729.83 \pm 0.46$ & $728.58 \pm 0.45$ & $729.08 \pm 0.85$ & $726.62 \pm 0.95$ & $729.28 \pm 0.39$ & $728.19 \pm 0.52$ & $729.22 \pm 0.67$ & $726.84 \pm 0.35$ \\
\hline Humidity & $70.43 \pm 8.87$ & $90.21 \pm 2.13$ & $88.16 \pm 3.54$ & $51.17 \pm 11.63$ & $63.42 \pm 15.9$ & $90.95 \pm 1.30$ & $89.8 \pm 3.09$ & $56.38 \pm 10.97$ & $52.14 \pm 4.50$ \\
\hline
\end{tabular}

Table 1: Average values and deviation of T, P, H

We can observe that standard deviation of different atmospheric parameters changing significantly during the day, but at night the variation of standard deviation is minimum and we observe that the particles rate has a direct correlation whit these changes. The graphs represent variation of pressure and temperature of different days during several months. The small fluctuations of temperature is due to the location of the WCD. Variations are the result of the morning sun irradiating on the front of to the sensor and the afternoon shadows of the building. The real temperature $T(h)$ depends on latitude and climate of the region producing significant changes through out the year. These variations influence the values of the meteorological coefficients.

\section{Conclusions}

The system we designed has been operating from August 2018 until May 2019. These 5 months of data were processed continuously. The Corsika simulations predict that Quito could be an appropriate place to study $\mathrm{TeV}$ astronomy. We plan to continue with the operation of the 
WCD to obtain more data of astroparticles, temperature, pressure and humidity. The variation of temperature and pressure reflects the variable weather of Quito, typical of the Andean highlands.

\section{References}

[1] S.Ostapchenko, PRD 83 (2011) 014018

[2] Castellina, A and Fulgione, W and , P.L.Ghia and Gomez, Francesco and Aglietta, Marco and Morello, C and , A.Chiavassa and, G.Navarra and Vigorito, C and Genolini, Bernard and Nguyen Trung, T and Benettin, G and Pennella, A Pierre Auger Observatory - SD : TEST BENCH AND TESTING PROCEDURES FOR PMT XP1805 / DI BASE PRODUCTION,

[3] D. Pavel, Red Pitaya Notes, [Online]. Available: http://pavel-demin.github.io/red-pitaya-notes

[4] I. Standards, C. Committee, D. Generation, and E. Storage, IEEE Guide for Array and Battery Sizing in Stand-Alone Photovoltaic (PV) Systems, no. May. 2008.

[5] C. Rosero, J. Cando, Diseño e implementación de una estación autónoma de monitoreo de rayos cósmicos y variables ambientales., Julio, 2018 [Online]. Available: https://bibdigital.epn.edu.ec/bitstream/15000/19588/1/CD-8985.pdf

[6] J. Poirier, T. Catanach, Atmospheric Effects on Muon Flux at Project GRAND, January 2011, doi:10.7529/ICRC2011/V11/0307

[7] L. Dorman Cosmic Rays in the Earth's Atmosphere and Underground, 2004, ISBN: 9781402020711 